\title{
SECAS METEOROLÓGICA, AGRÍCOLA, HIDROLÓGICA NO MUNICÍPIO DE SERRINHA-BA
}

\author{
SANTOS, Maria Auxiliadora Freitas - dorafreitas2004@yahoo.com.br \\ Universidade Federal do Pernambuco / UFPE
}

COSTA, Valéria Sandra de Oliveira - costavso@yahoo.com.br

Universidade Federal do Pernambuco / UFPE

GALVINCIO, Josiclêda Domiciano - josicleda@gmail.com

Universidade Federal do Pernambuco / UFPE

Submetido em: 27/05/2020

Aceito para publicação em: 14/05/2021

Publicado em: 01/06/2021

DOI: http://dx.doi.org/10.5380/abclima.v28i0.74084

\begin{abstract}
RESUMO: Compreender os diferentes tipos de seca constitui elemento importante no desenvolvimento de modelos para uma atuação proativa em diferentes cenários ao nível global. Assim, este artigo tem como objetivo analisar as consequências socioambientais dos diferentes tipos de secas no período de 1987 a 2018 no município de Serrinha, Bahia. Para determinar a intensidade das secas utilizou-se a série climatológica que compreende o período entre 1987 a 2018 e o Standardized Precipitation Index (SPI) nas escalas temporais de 3, 6, 9, 12 e 24 meses. A partir da classificação dos SPI's foram registrados 17 anos secos, com destaque para as categorias que variavam entre seca e extrema. Os anos de 1993 e 2012 foram registrados em todos os SPI's estudados, apresentando-se como os mais secos da série. Quanto às décadas, verifica-se que as de 1990 e 2010 apresentam uma maior quantidade de eventos secos em comparação à de 2000. O período estudado possibilitou a classificação das secas, sua duração e frequência bem como as consequências ambientais e econômicas na produção do milho, sendo esta cultura característica na região. Desta forma, ao conhecer a dinâmica das secas no município, ampliam-se as possibilidades de realizar e efetivar o planejamento voltado à convivência com a seca.
\end{abstract}

PALAVRAS-CHAVE: Tecnologias sociais, SPI, água.

\section{METEOROLOGICAL, AGRICULTURAL, HYDROLOGICAL DROUGHTS IN THE MUNICIPALITY OF SERRINHA-BA}

ABSTRACT: Understanding the different types of drought it is important element in the development of models for proactive action in different scenarios at the global level. Thus, this article has the means to analyze the socioenvironmental consequences of different types of droughts in the period from 1987 to 2018 in the municipality in Serrinha, located in the State of Bahia. To determine the intensity of droughts, the climatological series comprising the period from 1987 to 2018 and the Standardized Precipitation Index (SPI) were used in the 3, 6, 9, 12 and 24 month time scales. From the classification of the SPI's, 17 dry years were recorded, with emphasis on the categories that varied between drought and extreme. The years 1993 and 2012 were recorded in all studied SPI's, presenting themselves as the driest in the series. As for the decades, it can be seen that the 90 s and 10 s have a greater number of dry events compared to 2000. The period studied made it possible to classify the droughts; its duration and frequency, as well as the environmental and economic consequences in the production of corn, this crop being characteristic in the region. Thus, by knowing the dynamics of droughts in the municipality, they expand the possibilities of carrying out and carrying out planning aimed at living with drought.

KEYWORDS: Social technologies, SPI, water

SEQUIA METEOROLOGICAS, AGRICULTURALES, HIDROLOGICAS EN SERRINHA-BA 
RESUMEN : Comprender los diferentes tipos de sequía es un elemento importante en el desarrollo de modelos de acción proactiva en diferentes escenarios a nivel global. Este artículo cuenta con los medios para analizar las consecuencias socioambientales de diferentes tipos de sequías en el período de 1987 a 2018 en la municipalidad de Serrinha, ubicado en el Estado de Bahía. Para determinar la intensidad de las sequías se utilizó la serie climatológica que comprende el período de 1987 a 2018 y el Índice de Precipitación Estandarizado (SPI) en las escalas de tiempo de 3, 6, 9, 12 y 24 meses. A partir de la clasificación de los SPI's se registraron 17 años secos, con énfasis en las categorías que variaban entre sequía y extrema. Los años 1993 y 2012 se registraron en todos los SPI's estudiados, presentándose como los más secos de la serie. En cuanto a las décadas, se puede observar que las de 90 y 10 tienen un mayor número de eventos secos en comparación con 2000. El período estudiado permitió clasificar las sequías; su duración y frecuencia, así como las consecuencias ambientales y económicas en la producción de maíz, siendo este cultivo característico de la región. Así, al conocer la dinámica de las sequías en el municipio, amplían las posibilidades de realizar y llevar a cabo una planificación orientada a convivir con la sequía.

PALABRAS CLAVE: Tecnologíassociales, SPI, agua.

\section{INTRODUÇÃO}

A seca consiste em um fenômeno natural que pode afetar as condições sociais e econômicas de um lugar. Ela está relacionada à deficiência de precipitação, que, quando estendido por uma temporada ou longo período de tempo, resulta na incapacidade de atender às demandas socioambientais (HAYES et al., 2011). Diferentemente da aridez, a qual é uma característica permanente, a seca é um estado transitório (RAVELO; SANZ RAMOS; DOURIET CÁRDENAS, 2014).

Os pesquisadores Lonita, Scholz e Chelcea (2016) citam que a seca pode ser visualizada sob diferentes maneiras a depender dos usuários de água, as quais suas definições podem ser classificadas em dois tipos: conceitual e operacional. A primeira possibilita compreender o significado da seca e seus efeitos, no entanto, não fornecem dados quantitativos sobre quando, frequência e gravidade. Já a operacional auxilia na identificação do seu início, fim e grau de severidade.

Keyantash e Dracup (2002) utilizam a seguinte classificação para avaliar os índices de seca: meteorológica, hidrológica, agrícola e socioeconômica, sendo esta última consequência das demais, a qual pode ser obtida, dentre outras maneiras, a partir do índice monetário para sua avaliação. Também, estes mesmos autores, retratam a importância de seis critérios para o estabelecimento de índices necessários para avaliar as secas, dentre eles: robustez, tratabilidade, transparência, sofisticação, extensibilidade e dimensionabilidade. Cada um dos tipos de seca apresenta suas características em relação a duração e os impactos causados.

Esta classificação é importante, por exemplo, para relacionar a seca com questões voltadas à segurança alimentar, como revelam os estudos realizados por Hameed, Ahmadalipour e Moradkhani (2019), em que fizeram esta análise com as secas meteorológicas, agrícolas e hidrológicas em diferentes escalas de tempo no Oriente Médio e verificaram que a hidrológica é a mais intensa na região, especialmente no Egito, atingindo seu ápice em 2009. A seca meteorológica atingiu seu máximo no início dos anos 1970 e 2000 e entre 20082012, já a seca agrícola teve seu máximo em 2017, afetando $80 \%$ de extensão da região. Assim, o índice de produção agrícola obteve uma tendência 
decrescente durante o período de seca entre 2007 - 2013, enquanto o déficit alimentar cresceu nesta mesma época.

Ao estudar as secas e seus impactos, uma variedade de índices, incluindo diferentes variáveis meteorológicas, pode ser utilizada. Dentre eles: Índice de Severidade de Seca de Palmer (PDSI) (PALMER, 1965) que tem como base a evapotranspiração, a precipitação mensal e o balanço; Índice de Recuperação de Seca (RDI) que tem escala mensal e pode ser utilizado para a classificação da seca hidrológica (Weghorst, 1996); Índice de Seca de Bhalme \& Mooley (Bhalme \& Mooley Drought Index -BMDI) utilizando dados de precipitação e eficaz para regiões tropicais (BHALME; MOOLEY, 1980); e o Índice de Anomalia de Chuva (IAC) proposto por Van-rooy (1965).

Pode-se evidenciar também o Índice Padronizado de Precipitação (Standardized Precipitation Index, SPI) desenvolvido por McKee, Doeskene e Kleist (1993) como uma ferramenta conhecida capaz de quantificar e monitorar a seca em diferentes escalas de tempo (BLAIN; BRUNINI, 2005). O mesmo tem sido muito utilizado no monitoramento de seca em todo o mundo, inclusive recomendado pela Organização Meteorológica Mundial devido a sua flexibilidade (SUBASH; MOHAN, 2011).

Nascimento, Braga e Araújo (2017) ao analisarem eventos extremos de chuva e seca, utilizando o SPI, de acordo com sua frequência e período para os anos de 1987 e 2015 no Estado do Maranhão, verificaram que ocorrem duas estações do ano bem definidas quanto ao regime das chuvas. Para SPI 6, o qual foi utilizado nesta pesquisa, a maior escala de eventos secos ocorreu na década de 1990, coincidindo com o surgimento do El Nino, com destaque para a região Norte do estado.

Verificando as mudanças no padrão espaço-temporal das secas no Nordeste do Brasil, Cunha et al. (2018) identificaram que os maiores eventos de seca foram nos anos de 1982-1983, 1992-1993, 1997-1998, 2007-2008 e 2012-2016. Dentre estes, registram-se os anos entre 2012 -2016 como a seca mais intensa nas últimas décadas no tocante à intensidade.

Entre as secas registradas acima, Costa (2012) cita a sua relação com o fenômeno do El Nino registrado nos anos de 1877, 1982, 1983 e de 1997 em decorrência à modificação anômala durante a circulação atmosférica superior e alteração no movimento dos centros de altas e baixas pressões.

Quanto ao nordeste brasileiro, analisando as políticas públicas de secas, Campos (2014) ressalta que estas iniciaram neste local após o reconhecimento do governo em procurar meios para solucioná-la a partir da repercussão mundial das grandes secas de 1877 a 1879, em que morreram milhares de pessoas.

Este mesmo autor considerou, em seu trabalho, uma proposta de periodização da seca entre 1653 até 2012, classificando nos seguintes períodos: defrontamento com as secas (1583-1848); busca do conhecimento (18491877), hidráulica da solução (1877-1958), política do desenvolvimento em bases regionais (1959-1991); e gestão da água e o desenvolvimento (1992).

No semiárido nordestino, por exemplo, o fenômeno El Niño - Oscilação Sul (ENOS) apresenta uma relação de causa e efeito com as secas ocorrendo redução das chuvas. O El Nino 1982/1983, depois de três anos com baixa 
precipitação, levou a uma das maiores secas nesta região (SANTOS; CÂMARA, 2002).

Nesta perspectiva, Matos (2012) reflete que o enfrentamento para os efeitos da estiagem ocorre a partir de medidas governamentais paliativas e emergenciais e que no período entre 1950 e 1970 emergiu o planejamento a partir da visão tecnicista e cientificista, bem como o desenvolvimento por meio de ações desenvolvidas pela Comissão do Vale do São Francisco (CODEVASF), Banco do Nordeste (BNB), Superintendência de Desenvolvimento do Nordeste (SUDENE) e Grupo de Trabalho para o Desenvolvimento do Nordeste (GTDN).

Analisar e compreender a dinâmica das secas constitui elemento de extrema relevância em diferentes escalas. Estudos desenvolvidos ressaltam a forma como a seca afeta a produção agrícola, bem como desencadeamento de conflitos humanos negativos. Assim, urge a necessidade de monitorar seus eventos, bem como identificar sua frequência e identidade como uma das estratégias para pesquisas e implementação de cenários futuros (HAILE et al., 2019; HAILE et al., 2020; SUN et al., 2019).

Ao considerar a seca social, sob as perspectivas das políticas voltadas as ações para este fenômeno, Camurça et al. (2016) ao discutirem as implicações psicossociais da seca em uma comunidade rural no interior do Ceará, ressaltam que a relação entre a seca e falta de água possibilitaram a construção de grandes obras para armazenamento dos recursos hídricos, os quais eram restritivos aos detentores do poder, reforçando as premissas voltadas à indústria da seca. Desta forma, do período colonial até a atualidade, esta visão reducionista voltada à problemática associada à falta da água possibilita uma aceitação das consequências da seca enquanto problemas naturais.

Destaque também para as implicações da seca social nas questões voltadas à saúde pública conforme revelam os estudos realizados por Salvador et al. (2020) que citam questões voltadas a ampliação do suicídio entre agricultores do sexo masculino que residem em áreas rurais e possuem idade entre 30-49 anos durante eventos de seca na Austrália entre 1970-2007.

Desta forma, como uma das estratégias para desmistificar e superar o determinismo e as cadeias de naturalização impostas quanto ao fenômeno da seca, faz-se necessário analisar sua história, características físicas, sociais e econômicas e as novas possibilidades de políticas públicas que fomentem uma nova percepção, as quais podem ser fundamentadas para a convivência a partir da implementação de tecnologias sociais voltadas à captação e armazenamento da água de chuva.

Compreender os tipos de secas constituem elementos importantes no desenvolvimento de modelos para atuação proativa em diferentes cenários de seca globais (MISHRA; SINGH, 2010), pois afetam os recursos hídricos superficiais e subterrâneos, redução da sua quantidade e qualidade, bem como atividades produtivas e outras atividades econômicas e sociais.

Essa importância, também pode ser relacionada aos diferentes níveis, dentre eles, o regional e o local, vide exemplo o município de Serrinha-BA, onde foi realizado este estudo, que está situado no semiárido nordestino brasileiro e tem a agricultura familiar como uma das bases para a economia. Ao qual se justifica pela necessidade de conhecer as características das secas nesta localidade como uma das estratégias para propor políticas públicas que possam 
se adequar às secas e situações sociais, ambientais e econômicas, de forma a contribuir com ações que visem o desenvolvimento local a partir das suas especificidades existentes e futuras.

Desta forma, este trabalho tem como objetivo analisar as consequências socioambientais dos diferentes tipos de secas no período de 1987 a 2018 no município de Serrinha-BA.

\section{MATERIAL E MÉTODOS ÁREA EM ESTUDO}

O lócus desta pesquisa é um município localizado no Estado da Bahia, denominado Serrinha, inserido no Território do Sisal, conforme destaque na Figura 1. Este município além de ser o local central em que tem a entidade executora dos programas voltados a implementação das tecnologias sociais voltadas à captação e utilização da água de chuva, vide exemplo, cisterna calçadão e barragem subterrânea, é um pólo estratégico que concentra diversos serviços dentre os vinte municípios do território do sisal. Serrinha possui uma população total de 80.861 pessoas, em que a porcentagem do urbano perfaz $61,5 \%$ e a rural $38.5 \%$ (IBGE, 2010). Com atividades econômicas, para o ano de 2016, associadas aos setores de agropecuária $(1,7 \%)$, indústria $(12,7 \%)$, Comércio e Serviços (85,6\%) (IBGE, 2018; SEI, 2018).

Em relação à Região de Planejamento de Gestão das Águas (SEI-BA, 2003), este município encontra-se entre as RPGA's do Recôncavo Norte 2 e Paraguaçu; tem como bioma predominante, a Caatinga; o solo apresenta característica, em sua maior parte, de Planossoloseutróficos. Com um total de 3.803 unidades de estabelecimentos da agricultura familiar nesta localidade (PTDSS, 2016).

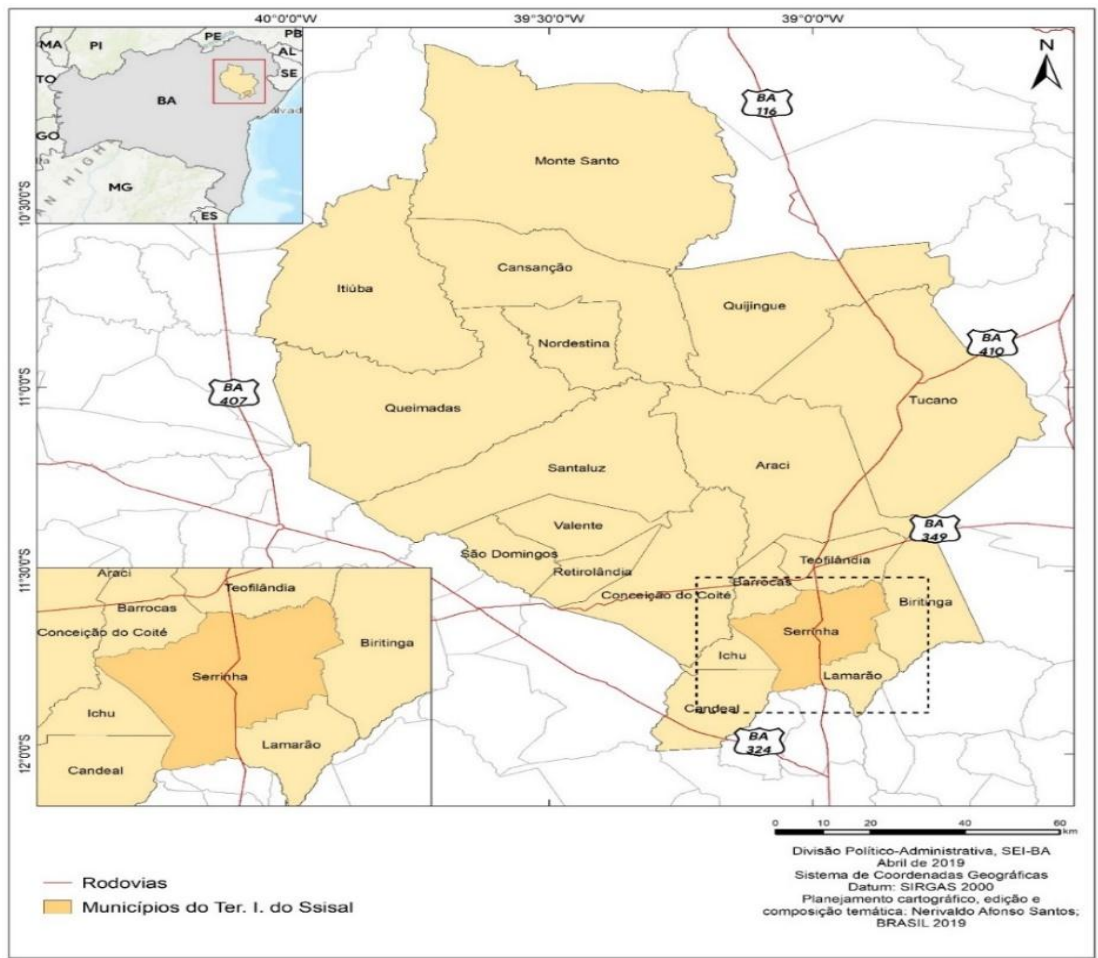

Figura 1- Delimitação do município de Serrinha-BA. Fonte: Elaborado a partir dos dados de SEI-BA (2019). 


\section{MÉTODOS}

Para a determinação das intensidades das secas utilizou-se o Standardized Precipitation Index (SPI) nas escalas temporais de 1,3, 6, 12 e 24 meses (MCKEE; DOESKEN; KLEIST, 1993), em que quantifica o déficit de precipitação em várias escalas de tempo, podendo monitorar períodos úmidos e secos. O SPI utiliza apenas os dados mensais de precipitação, estes são normalizados de modo que os climas mais úmidos e secos sejam representados de uma maneira similar.

Para realizar o cálculo do SPI, primeiro foi determinada a probabilidade da distribuição da precipitação pluvial, sendo calculada através da distribuição de probabilidade Gama conforme a equação 1:

$$
g(x)=\frac{1}{\beta a t(a)} x^{a-1} e^{-x} / \beta
$$

Em que:

$a>0=$ parâmetro da distribuição gama; $\beta>0=$ Parâmetro de escala de distribuição gama; $x>0$ é a quantidade de precipitação $(\mathrm{mm})$.

A função gama foi calculada com base na equação 2 :

$$
\Gamma(\alpha)=\int_{-0}^{\infty} \cdot \mathrm{Y}^{\mathrm{a}-1} \mathrm{e}^{-\mathrm{Y}} \mathrm{dy}
$$

Os parâmetros a e $\beta$ da função densidade de probabilidade gama, foram estimados para cada estação de acordo com as escalas de tempo que interessam para o estudo e para cada mês do ano. Foram usadas soluções de máxima verosimilhança para determinar a e $\beta$, conforme equações 3 e 4 .

$$
\begin{aligned}
& \alpha=\frac{1}{4 A}\left(1+\sqrt{1+\frac{4 A}{3}}\right. \\
& \beta=\frac{\bar{x}}{\alpha}
\end{aligned}
$$

Sendo que: $\mathrm{A}==\ln (\bar{x})-\sum_{i=1}^{N} \ln (x)$

$\mathrm{E}$ que: $\bar{x}$ é a média aritmética da precipitação $(\mathrm{mm}) ; \mathrm{N}$ é o número de observações de precipitação; ln é o logaritmo neperiano.

Foram utilizados também os parâmetros para estimar a probabilidade cumulativa de um evento de precipitação na escala de tempo desejada. Assim, a probabilidade cumulativa é dada por:

$$
G(x)=\int_{0}^{x} g(x) d x=\frac{1}{\beta \alpha \Gamma(\mathrm{a})} \int_{0}^{x} x^{\alpha-1} e-x / \beta d x
$$

Organizando $t=x / \beta$, a equação 6 transforma-se na função gama incompleta:

$$
G(x)=\frac{1}{\Gamma(\mathrm{a})} \int_{0}^{x} t^{\alpha-1} e^{-t} \mathrm{dt}
$$

Como a função gama $\Gamma(\alpha)$ não esta definida para $\mathrm{x}=0$ e a amostra pode ter zeros, a probabilidade cumulativa é expressa por: 


$$
H(x)=q+(1-q) G(x)
$$

Sendo: $H(x)$ é a distribuição de probabilidade cumulativa; q é a probabilidade de ocorrência de valores nulos (zeros); $\mathrm{G}(\mathrm{x}$ ) é a distribuição cumulativa teórica. que: Caso $\mathrm{m}$ seja o número de zeros em uma série de precipitação, ocorre

$$
\mathrm{q}=\frac{m}{n+1}
$$

onde $m \rightarrow$ número de ordem dos valores de zero em uma série climatológica $n \rightarrow$ tamanho da amostra

Em seguida, o valor de (Z) ou SPI foi obtido pela aproximação matemática desenvolvida por Abramowitz e Stegun (1965), que converte a probabilidade cumulativa em uma distribuição normal a variável (Z).

$$
\begin{aligned}
& Z=S P I=-\left(t-\frac{c_{0}+c_{1} t+c_{2} t^{2}}{1+d_{1} t+d_{2} t^{2}+d_{3} t^{3}}\right) \text { para } 0<H(x) \leq 0.5 \\
& Z=S P I=+\left(t-t-\frac{c_{0}+c_{1} t+c_{2} t^{2}}{1+d_{1} t+d_{2} t^{2}+d_{3} t^{3}}\right) \text { para } 0.5<H(x) \leq 1.0 \\
& \text { Onde: } t=\sqrt{\ln \left(\frac{1}{(H(x))^{2}}\right)} \text { para } 0<H(x) \leq 0.5 \\
& t=\sqrt{\ln \left(\frac{1}{1-(H(x))^{2}}\right)} \text { para } 0.5<H(x) \leq 1.0
\end{aligned}
$$

Os coeficientes usados nas equações 12 e 13 foram: $c_{1}=0,802853 ; c_{2}$ $=0,010328 ; d_{1}=1,432788 ; d_{2}=0,189269 ; d_{3}=0,001308$.

O valor para calcular o SPI inicia a partir do ajuste da função densidade de probabilidade Gama às séries de dados mensais das precipitações, sendo estimada a probabilidade acumulada da frequência de cada total mensal (FARIAS; SOUZA; SOUSA, 2014).

A presença ou não de seca, bem como seu grau de intensidade com o uso do SPI foram definidos de acordo com MCkee, Doesken e Kleist (1993). Se o valor do SPI é continuamente negativo, com um valor de intensidade igual ou menor que menos um, a seca é evidente. E termina quando o valor do SPI apresenta-se como positivo. Esta classificação é realizada conforme os limites da Tabela 1, em que é possível caracterizar secas e períodos mais úmidos.

Tabela 1- Valores do Índice Padronizado de Precipitação (SPI) e categorias de períodos secos e úmidos.

Fonte: McKee, Doesken e Kleist (1993).

\begin{tabular}{ll}
\hline Limites do SPI & Categorias \\
\hline 2,0 ou maior & Chuva extrema \\
1,50 a 1,99 & Chuva Severa \\
1,00 a 1,49 & Chuva Moderada \\
0 a 0,99 & Chuva Fraca \\
0 a $-0,99$ & Seca Fraca \\
$-1,00$ a $-1,49$ & Seca Moderada \\
1,50 a $-1,99$ & Seca Severa \\
$-2,0$ ou menor & Seca extrema \\
\hline
\end{tabular}


Como o SPI requer uma série climatológica com dados de no mínimo 30 anos, esta análise baseou-se no o período entre 1987 a 2018 em virtude ser este o espaço de tempo em que foram implementadas tecnologias sociais voltadas à captação da água de chuva e destinadas à produção agrícola no município de Serrinha, o que torna importante a compreensão sobre a dinâmica da seca local.

As bases de dados foram obtidas nos órgãos oficiais do Instituto Brasileiro de Geografia e Estatísticas (IBGE), do Instituto Nacional de Meteorologia (INMET), do Instituto do Meio Ambiente e Recursos Hídricos (INEMA), e do Sistema Eletrônico de Informações (SEI-BA).

Para este estudo foram definidas as escalas 1(SPI1), 3(SPI3), 6 (SPI6), 12 (SPI12) e 24 (SPI 24) meses. A série histórica utilizou dados históricos do BDMET- INMET da Estação Serrinha-BA (OMM: 83190), Latitude:- 11.63, Longitude ${ }^{\circ}$-38.96, Altitude: 359.63 m. Início da Operação: 01.02.1904, tendo 31 anos como base. E ainda considerados os SPI1 e 3 para classificar a seca em meteorológica, o SPI6 e 12 para a seca agrícola e o SPI24 para a hidrológica, com base na caracterização das secas do estudo de Silva, Santos e Pires (2005).

\section{REULTADOS E DISCUSSÃO}

Utilizando o SPI1 e 3 para caracterizar os anos mais secos a partir da classificação de seca meteorológica em uma série histórica de 31 anos, registrou-se segundo o SPI1, 17 eventos de seca, sendo 12 classificadas em severa e cinco em extrema, com duração variando de um a três meses. Os eventos das categorias secas extremas ocorreram nos períodos e em intensidades atingindo valores, respectivamente: out/93 $(-2,42)$, set/97 ($2,61)$, nov/09 $(-2,47)$, ago/17 $(-2,57)$ e jul/28 $(-3,04)$. Quanto às secas severas, o período de fev a abr/93 registrou a maior duração, três meses; e o de jan a fev/17, com dois meses (Tabela 2).

Quanto ao SPI3, foram registrados 11 eventos secos, sendo oito classificados em severos e três em extremos. Para as secas severas, a que ocorreu entre fev e jul/93 durou seis meses $(-1,82)$, entre jun a set/11, quatro meses $(-1,68)$, a de set/04 a out/04, bem como a de nov/15 a dez/2015, dois meses $(-1,76,-1,64$, respectivamente), as demais secas severas registraram um evento. Para as secas extremas, uma ocorreu entre out a nov/97 $(-2,12)$, com duração de dois meses, a de mar a jul/2012 $(-1,68)$, cinco meses, e set/18 $(-2,11)$, um mês de duração (Tabela 2$)$. 
Tabela 2 - SPI's, quantidade (eventos), período, SPI médio, duração (meses) e categoria das secas para o município de Serrinha-BA.

\begin{tabular}{|c|c|c|c|c|c|c|}
\hline \multirow{2}{*}{ SPI'S } & \multirow{2}{*}{ Quantidade } & \multicolumn{2}{|l|}{ Período } & \multirow{2}{*}{$\begin{array}{l}\text { SPI } \\
\text { Médio }\end{array}$} & \multirow{2}{*}{ Duração } & \multirow{2}{*}{ Categoria } \\
\hline & & Início & Fim & & & \\
\hline \multirow{17}{*}{ SPI1 } & 1 & jul/87 & jul/87 & $-1,7$ & 1 & Seca Severa \\
\hline & 2 & $\mathrm{fev} / 93$ & $\mathrm{abr} / 93$ & $-1,60$ & 3 & Seca Severa \\
\hline & 3 & out/93 & out/93 & $-2,42$ & 1 & Seca Extrema \\
\hline & 4 & mai/96 & mai/96 & $-1,69$ & 1 & Seca Severa \\
\hline & 5 & set/97 & set/97 & $-2,61$ & 1 & Seca Extrema \\
\hline & 6 & out/02 & out/02 & $-1,62$ & 1 & Seca Severa \\
\hline & 7 & set/04 & set/04 & $-1,61$ & 1 & Seca Severa \\
\hline & 8 & dez/04 & $\mathrm{dez} / 04$ & $-1,5$ & 1 & Seca Severa \\
\hline & 9 & mar/09 & mar/09 & $-1,64$ & 1 & Seca Severa \\
\hline & 10 & nov/09 & nov/09 & $-2,47$ & 1 & Seca Extrema \\
\hline & 11 & $\mathrm{abr} / 12$ & $\mathrm{abr} / 12$ & $-1,9$ & 1 & Seca Severa \\
\hline & 12 & nov/15 & nov/15 & $-1,93$ & 1 & Seca Severa \\
\hline & 13 & $\mathrm{mar} / 16$ & $\mathrm{mar} / 16$ & 1,63 & 1 & Seca Severa \\
\hline & 14 & jan/17 & fev/17 & $-1,77$ & 2 & Seca Severa \\
\hline & 15 & ago/17 & ago/17 & $-2,57$ & 1 & Seca Extrema \\
\hline & 16 & jan/18 & jan/18 & $-1,5$ & 1 & Seca Severa \\
\hline & 17 & jul/18 & jul/18 & $-3,04$ & 1 & Seca Extrema \\
\hline \multirow{11}{*}{ SPI3 } & 1 & set/87 & set/87 & $-1,62$ & 1 & Seca Severa \\
\hline & 2 & $\mathrm{fev} / 93$ & $\mathrm{jul} / 93$ & $-1,82$ & 6 & Seca Severa \\
\hline & 3 & out/95 & out/95 & $-1,83$ & 1 & Seca Severa \\
\hline & 4 & out/97 & nov/97 & $-2,12$ & 2 & Seca Extrema \\
\hline & 5 & set/04 & out/04 & $-1,76$ & 2 & Seca Severa \\
\hline & 6 & $\mathrm{fev} / 06$ & $\mathrm{fev} / 06$ & $-1,67$ & 1 & Seca Severa \\
\hline & 7 & jan/07 & jan/07 & $-1,5$ & 1 & Seca Severa \\
\hline & 8 & jun/11 & set/11 & $-1,68$ & 4 & Seca Severa \\
\hline & 9 & mar/12 & Jul/12 & $-2,07$ & 5 & Seca Extrema \\
\hline & 10 & nov/15 & dez/15 & $-1,64$ & 2 & Seca Severa \\
\hline & 11 & set/18 & set/18 & $-2,11$ & 1 & Seca Extrema \\
\hline \multirow{7}{*}{ SPI6 } & 1 & jan/93 & dez/93 & $-1,68$ & 12 & Seca Severa \\
\hline & 2 & nov/97 & nov/97 & $-1,82$ & 1 & Seca Severa \\
\hline & 3 & out/04 & out/04 & $-1,55$ & 1 & Seca Severa \\
\hline & 4 & out/04 & dez/04 & $-1,5$ & 3 & Seca Severa \\
\hline & 5 & set/11 & set/11 & $-1,72$ & 1 & Seca Severa \\
\hline & 6 & $\mathrm{abr} / 12$ & out/12 & $-2,05$ & 7 & Seca Extrema \\
\hline & 7 & $\mathrm{mai} / 17$ & jun/17 & $-1,59$ & 2 & Seca Severa \\
\hline \multirow{4}{*}{ SPI12 } & 1 & mar/93 & dez/93 & $-2,25$ & 10 & Seca Extrema \\
\hline & 2 & jan/95 & mar/95 & $-1,54$ & 3 & Seca Severa \\
\hline & 3 & mar/12 & abr/13 & $-1,8$ & 14 & Seca Severa \\
\hline & 4 & nov/17 & jan/18 & $-1,51$ & 3 & Seca Severa \\
\hline \multirow{5}{*}{ SPI24 } & 1 & fev/93 & dez/93 & $-1,94$ & 11 & Seca Severa \\
\hline & 2 & jan/95 & fev/96 & $-1,58$ & 13 & Seca Severa \\
\hline & 3 & out/12 & mai/14 & $-1,5$ & 20 & Seca Severa \\
\hline & 4 & jan/18 & fev/18 & $-1,8$ & 2 & Seca Severa \\
\hline & 5 & nov/18 & nov/18 & $-1,58$ & 1 & Seca Severa \\
\hline
\end{tabular}

Fonte: Elaborada a partir dos dados do INMET (2019).

Ao verificar os SPI's1 e 3, observa-se que seca severa apresenta uma maior quantidade de eventos e de duração em relação à extrema, com destaque para os anos 1987, 1993, 1997, 2012, 2015 e 2018 (Tabela 2). E ao analisar a climatologia do município de Serrinha-BA no período estudado, percebe-se que 
os anos de 1993 e 2012, os quais apareceram nos SPI's1 e 3, apresentaram no total, valores de precipitação abaixo das normais climáticas para o período. Quanto à série 1987-2018, o máximo de precipitação foi de $86,8 \mathrm{~mm}$ e mínimo de 35,5 mm. Para os anos de 1993, o máximo foi de $69,3 \mathrm{~mm}$ e mínimo de 0,7 $\mathrm{mm}$ e em 2012 o máximo foi de $57,1 \mathrm{~mm}$ e $0 \mathrm{~mm}$ em dezembro (Figura 2).

Ao analisar o surgimento das secas severas e extremas por décadas, com os SPI's1 e 3, para o SPI1, as cinco secas extremas apareceram em todas as décadas estudadas (1990, 2000 e 2010), enquanto para o SPI3, elas foram registradas em 1990 e 2010 (Tabela 2).

Nesta perspectiva, estudos de Guedes et al. (2016) também registraram secas em curtos espaços de tempo no Estado de Pernambuco para o ano de 2012 ao avaliar eventos de chuvas e secas utilizando o SPI na escala de três meses. Além deste ano, eles verificaram também 1993, 1998 e 2013 com os maiores déficits.

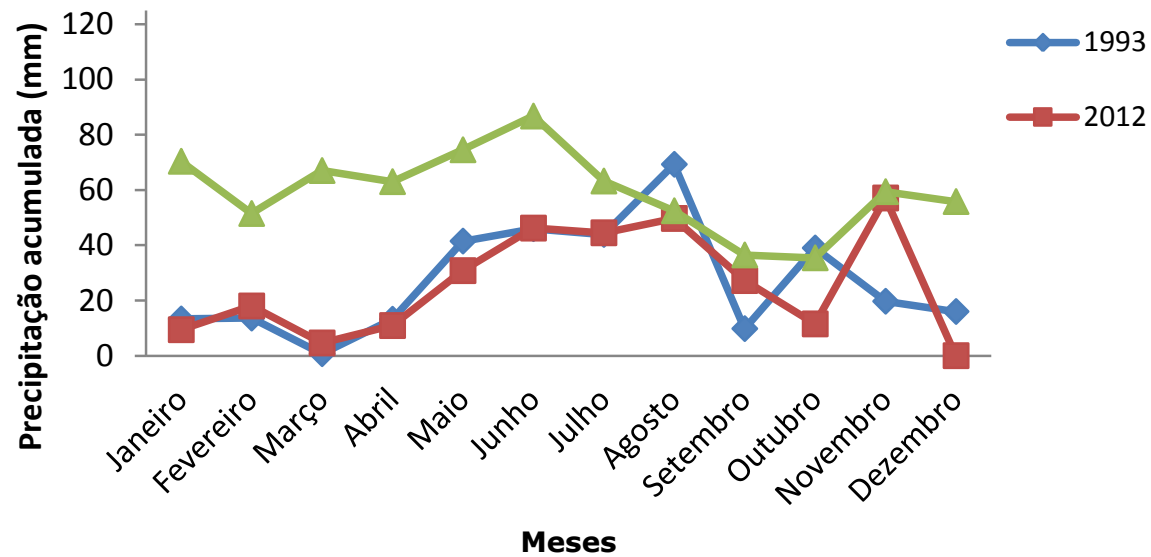

Figura 2 - Precipitação acumulada $(\mathrm{mm})$ no período de 1987-2018 e para os anos mais secos da série histórica, 1993 e 2013, para o município de Serrinha-BA. Estação: Serrinha (83190). Fonte: Elaborado a partir dos dados de INMET (2020).

Em relação à duração dos eventos para o SPI1, 15 deles tiveram a duração de um mês. Para o SPI3, variou de um a seis meses, com destaque para o ano em que era registrada uma duração maior, no outro período, a possibilidade de redução era mais evidente. Segundo análise de Edwards e Mckee (1997) quando as secas de longa duração ocorrem, podem ser acompanhadas de outras a curto prazo de forma consequente ou intercaladas a períodos curtos de umidade. $\mathrm{E}$ os principais impactos que podem ocorrer, considerando o SPI3, consistem em diminuir a produção e diversificação das culturas, bem como a geração de renda e possibilidade de gerar sobrecarga de trabalho (FARIAS; SOUZA; SOUSA, 2014).

Em janeiro de 2012 a seca intensificou-se no nordeste, sendo considerada a pior dos últimos 30 anos. Houve registros, na Bahia, de redução da precipitação a partir de maio de 2011, o que torna a região ainda mais vulnerável, incluindo o município de Serrinha-BA. (LEIVAS et al, 2012)

A classificação das secas desta série climatológica também contribui com a compreensão das dinâmicas de armazenamento das águas subterrâneas locais, assim como fez Haied et al. (2017) em seus estudos, ao monitorarem a 
severidade e duração da seca meteorológica na região WadiDjelfa, Sub-bacia do Hadjia, a partir do SPI, Seca de Reconhecimento (RDI) e Potencial de Evapotranspiração (PET) no período de 1975 a 2015, onde verificaram neste local quatro eventos de seca caracterizados em severidade moderada a extrema durante 1977-1978, 1983-1984, 1996-2003 e 2014-2015.

Nesta análise de SPI's1 e 3 e considerando como seca meteorológica no município em estudo, percebe-se sua importância na promoção de planejamento das atividades agrícolas-BA, tendo como base a realização de outros estudos, vide exemplo, o realizado por Oloruntade et al. (2017) em que ao estudarem índices de seca para comparação deste fenômeno na bacia do Níger-Sul, uma sub-bacia hidrográfica da bacia do Baixo Níger, utilizaram dentre eles, o SPI na avaliação da ocorrência de seca meteorológica durante o período 1970-2008 de forma a auxiliar no planejamento de tomada de decisões no tocante aos recursos hídricos, especialmente sob condições de mudança climática em uma escala de bacia.

Assim como também o estudo realizado em outras regiões do semiárido brasileiro, em que Buriti e Barbosa (2018) ao analisarem a duração, frequência e gravidade das secas meteorológicas revelaram os anos de 2000 a 2010 como a maior seca existente nesta região. Houve registro voltado a associação entre a seca e o fenômeno de El Niño, uma vez que, das 32 secas e dos 30 eventos do El Niño que ocorreram na área estudada, ocorreu associação em 23 casos estudados.

Considerando a série histórica para os SPI6 e 12 e classificando em secas agrícolas, para o SPI6, tem-se o registro de sete eventos secos, em que seis deles são classificados em seca severa e um em seca extrema. Em relação à sua duração, a seca severa que ocorreu entre jan e dez/93 teve a maior duração. Para o único evento de seca extrema, ela ocorreu entre abr a out/2012, duração de sete meses e pico de intensidade com valor de -2,05 (Tabela 2).

Com o SPI12, foram quatro eventos de seca, sendo três severos e um extremo. Com destaque para a seca severa que ocorreu entre mar/12 e abr/13 com duração de 14 meses e intensidade de $-1,8$. Quanto à seca extrema, entre mar e dez/93, houve duração de dez meses e intensidade $-2,25$. Os anos de 1993 e 2012 surgiram novamente nos SPI's6 e 12, assim como o ano de 2017. Com registro da seca extrema apenas na década de 2010, e no SPI12 na década de 1990. De acordo com o Monitor de secas (2021) esta classificação de seca pode ocasionar grandes perdas das culturas, das pastagens, bem como escassez ou restrição de água. Quanto à utilização do SPI24, classificando a seca em hidrológica, houve registro de cinco eventos secos, todos classificados em secas severas, ocorrendo entre fev a dez/93; jan/95 a fev/96; out/12 a maio/14, com maior duração (20 meses) e intensidade de $-1,5$; jan a fev/18; e nov/18, não sendo verificada a seca extrema (Tabela 2).

Ao ampliar as escalas do SPI, ocorreu, neste estudo, uma redução do número de eventos de seca e aumento da sua duração, em que o SPI1 foram 17 eventos, SPI3 (11), SPI9 (4), SPI12 (4) e SPI24 (5), verificando-se que índices mais longos necessitam de maior quantidade de tempo para ocorrer a recuperação. Quanto à esta questão, Chao et al. (2016), no sudoeste da China, verificaram uma variação aproximada de 17 meses de duração da seca, a qual foi caracterizada em hidrológica. A partir do satélite, percebeu-se que as secas 
sempre foram acompanhadas de precipitação anormal negativa, sendo diretamente associadas à precipitação.

Souza et al. (2018) consideram que o gerenciamento de riscos para secas e enchentes, em que utiliza a variável umidade do solo, constitui um importante elemento para estabelecer a severidade da seca, uma vez que este parâmetro se encontra relacionado ao ciclo hidrológico, fornecendo informações sobre impactos ecológicos, ambientais e agrícolas.

Conforme a Tabela 2, algumas secas que foram registradas no SPI1, não aparecem nos SPI's3, 6, 12 e 24, a exemplo da seca severa que ocorreu em mar/2016, bem como a mudança de categorias entre severa e extrema de algumas delas. Sob esta questão, Macedo, Guedes e Sousa (2011) verificaram também, ao estudar as secas e chuvas em Campina Grande-PB que àquelas detectadas no SPI3 não permaneceram no SPI6 o que pode ser justificado pelo surgimento de precipitação em um período maior, sendo que as secas que ficaram nas escalas ampliadas tiveram como consequências maiores impactos da disponibilidade hídrica local. Neste estudo pode-se perceber esta situação com as secas que ocorreram nos anos de 1996, 2002, 2009, 2016 e 2017.

No município de Serrinha, avaliando as décadas e os SPI's estudados, registraram-se eventos de secas em todas as décadas, no entanto a sua categoria, bem como os eventos de umidade vão sendo modificados. Conforme a Tabela 3, a década de 2010 registrou um percentual maior de eventos secos em relação às décadas de 1990 e 2000 e menor de eventos úmidos em relação à década de 2000. Assim verifica-se que os eventos secos foram mais frequentes nas décadas de 1990 e 2010. E a década de 2000 apresentou-se com maior teor de umidade, em que, ao utilizar o SPI 24, não foram registrados eventos de seca nas categorias moderadamente, muito e extremamente seco.

Nesta situação, as categorias de muito e extremamente seco foram mais frequentes nas décadas de 1990 e 2010, sendo na década de 2000, a depender do SPI aqui estudado, não houve registro das mesmas. Assim, a partir da análise de períodos secos e úmidos e considerando o fenômeno enquanto cíclico, o poder público municipal pode realizar estratégias públicas que possibilitem associar a captação e armazenamento da água de chuva em períodos mais chuvosos para posterior utilização em períodos mais secos a partir da implementação de tecnologias sociais associadas a esta finalidade.

Tabela 3 - Frequência decadal (\%) dos eventos nas categorias secos e úmidos obtidos pelo SPI's no município Serrinha-BA.

\begin{tabular}{llllll}
\hline SPI's & Categorias & $\mathbf{1 9 9 0}$ & $\mathbf{2 0 0 0}$ & $\mathbf{2 0 1 0}$ & Total \\
\hline & Pouco Seco (\%) & 18,06 & 22,22 & 22,22 & 62,50 \\
& Moderadamente Seco (\%) & 5,56 & 6,94 & 9,03 & 21,53 \\
& Muito Seco (\%) & 1,39 & 3,47 & 4,86 & 9,72 \\
& Extremamente Seco (\%) & 2,08 & 2,08 & 2,08 & 6,25 \\
\multirow{3}{*}{ SPI1 } & Total (\%) & $\mathbf{2 7 , 0 8}$ & $\mathbf{3 4 , 7 2}$ & $\mathbf{3 8 , 1 9}$ & $\mathbf{1 0 0 , 0 0}$ \\
& Pouco Úmido (\%) & 14,38 & $\mathbf{2 8 , 7 5}$ & 25,00 & 70,63 \\
& Moderadamente Úmido (\%) & 4,38 & 8,75 & 5,63 & 63,83 \\
& Muito Úmido (\%) & 0,63 & 6,25 & 0,63 & 25,53 \\
& Extremamente Úmido (\%) & 1,25 & 0,00 & 1,88 & 10,64 \\
& Total (\%) & $\mathbf{2 0 , 6 3}$ & $\mathbf{4 3 , 7 5}$ & $\mathbf{3 3 , 1 3}$ & $\mathbf{1 0 0 , 0 0}$ \\
\hline SPI3 & Pouco Seco (\%) & 16,67 & 27,56 & 25,64 & 69,87 \\
\hline
\end{tabular}




\begin{tabular}{|c|c|c|c|c|c|}
\hline & Moderadamente Seco (\%) & 3,21 & 3,85 & 8,33 & 15,38 \\
\hline & Muito Seco $(\%)$ & 3,21 & 2,56 & 3,85 & 9,62 \\
\hline & Extremamente Seco (\%) & 1,92 & 0,00 & 3,21 & 5,13 \\
\hline & Total $(\%)$ & 25,00 & 33,97 & 41,03 & 100,00 \\
\hline & Pouco Úmido (\%) & 15,97 & 29,17 & 25,69 & 70,83 \\
\hline & Moderadamente Úmido (\%) & 3,47 & 9,03 & 4,17 & 16,67 \\
\hline & Muito Úmido (\%) & 1,39 & 6,25 & 0,00 & 7,64 \\
\hline & Extremamente Úmido (\%) & 2,08 & 2,08 & 0,69 & 4,86 \\
\hline & Total (\%) & 22,92 & 46,53 & 30,56 & 100,00 \\
\hline \multirow{10}{*}{ SPI6 } & Pouco Seco $(\%)$ & 18,35 & 22,78 & 29,11 & 70,25 \\
\hline & Moderadamente Seco (\%) & 4,43 & 3,16 & 8,86 & 16,46 \\
\hline & Muito Seco $(\%)$ & 3,80 & 1,27 & 3,80 & 8,86 \\
\hline & Extremamente Seco (\%) & 1,90 & 0,00 & 2,53 & 4,43 \\
\hline & Total $(\%)$ & 28,48 & 27,22 & 44,30 & 100,00 \\
\hline & Pouco Úmido (\%) & 11,27 & 35,21 & 21,13 & 67,61 \\
\hline & Moderadamente Úmido (\%) & 2,11 & 9,86 & 4,93 & 16,90 \\
\hline & Muito Úmido (\%) & 2,82 & 8,45 & 0,00 & 11,27 \\
\hline & Extremamente Úmido (\%) & 2,82 & 0,70 & 0,70 & 4,23 \\
\hline & Total $(\%)$ & 19,01 & 54,23 & 26,76 & 100,00 \\
\hline \multirow{10}{*}{ SPI12 } & Pouco Seco $(\%)$ & 21,30 & 20,71 & 30,77 & 72,78 \\
\hline & Moderadamente Seco (\%) & 1,78 & 1,78 & 8,88 & 12,43 \\
\hline & Muito Seco $(\%)$ & 0,00 & 0,00 & 7,10 & 7,10 \\
\hline & Extremamente Seco (\%) & 5,92 & 0,00 & 1,78 & 7,69 \\
\hline & Total $(\%)$ & 28,99 & 22,49 & 48,52 & 100,00 \\
\hline & Pouco Úmido (\%) & 8,40 & 45,04 & 15,27 & 68,70 \\
\hline & Moderadamente Úmido (\%) & 3,05 & 15,27 & 4,58 & 22,90 \\
\hline & Muito Úmido (\%) & 6,11 & 2,29 & 0,00 & 8,40 \\
\hline & Extremamente Úmido (\%) & 0,00 & 0,00 & 0,00 & 0,00 \\
\hline & Total (\%) & 17,56 & 62,60 & 19,85 & 100,00 \\
\hline \multirow{10}{*}{ SPI24 } & Pouco Seco $(\%)$ & 11,31 & 16,07 & 29,17 & 56,55 \\
\hline & Moderadamente Seco (\%) & 2,38 & 0,00 & 14,88 & 17,26 \\
\hline & Muito Seco (\%) & 10,12 & 0,00 & 13,69 & 23,81 \\
\hline & Extremamente Seco (\%) & 2,38 & 0,00 & 0,00 & 2,38 \\
\hline & Total $(\%)$ & 26,19 & 16,07 & 57,74 & 100,00 \\
\hline & Pouco Úmido (\%) & 7,04 & 50,00 & 14,79 & 71,83 \\
\hline & Moderadamente Úmido (\%) & 12,68 & 12,68 & 0,00 & 25,35 \\
\hline & Muito Úmido (\%) & 0,00 & 2,82 & 0,00 & 2,82 \\
\hline & Extremamente Úmido (\%) & 0,00 & 0,00 & 0,00 & 0,00 \\
\hline & Total (\%) & 19,72 & 65,49 & 14,79 & 100,00 \\
\hline
\end{tabular}

Fonte: Elaborada a partir dos dados do INMET (2019).

Ao analisar os anos estudados em relação à extensão das secas e sua frequência nas escalas dos SPI's, pode-se verificar que os anos de 1993 e 2012 apareceram em todas as escalas, sendo as secas caracterizadas, neste período, em meteorológica, agrícola e hidrológica (Figura 3). E ao associar aos eventos de El Nino e La Niña, a seca de 2012 no nordeste, a qual pode ser estendida à localidade estudada, Marengo, Cunha e Alves (2016) citam que entre as secas mais recentes, as que ocorreram em 1998, 2002 e 2015 estão relacionadas ao evento do El Nino e que apesar de pesquisas revelarem que o Nordeste brasileiro apresenta maiores episódios de chuva nos eventos de La Niña, no entanto o ano de 2012 teve um comportamento 
diferente em que ocorreram inundações no leste da Amazônia e uma das mais graves secas no Nordeste brasileiro.

Quanto à seca que ocorreu em 1993, classificada em severa e extrema a depender do SPI, verifica-se que esta ocorreu em um período de El Niño (BURITI; BARBOSA, 2018) e evidenciada pelo baixo índice pluviométrico deste ano em relação aos demais da série, bem como aparece em todos as classificações estudadas. Barbosa e Laksmi Kumar (2016) citam estudos que retratam as modificações das categorias de secas e das anomalias de precipitação no Nordeste associadas ao El Ninõ, Oscilação Decadal do Pacifico e Oscilação Multidecadal do Atlântico.

Ao analisar a série Climatologia entre 1987 a 2011, percebe-se que anteriormente ao ano de 2011 , os anos secos não surgiam de forma sequencial. A partir de 2011 até 2018, registram-se eventos frequentes de secas em todos os anos, evidenciando as secas meteorológica, agrícola e hidrológica.

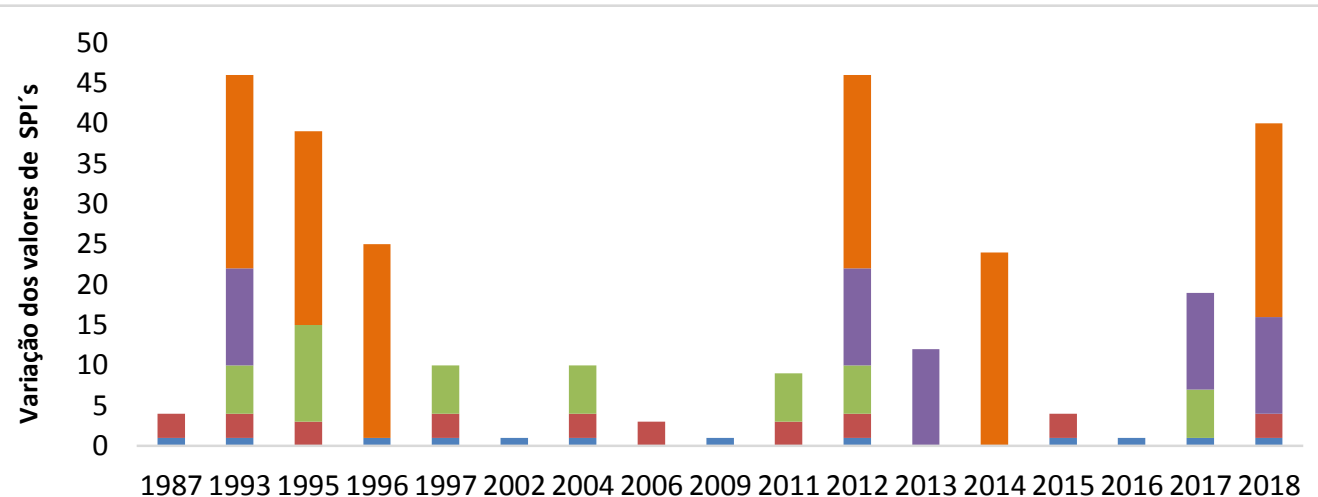

Anos

-SPI 1 SPI 3 SPI 6 SPI 12 SPI 24

Figura 3 - Categorias de SPI's por ano seco na série climatológica 1987 a 2018 no município de Serrinha-BA. Fonte: Elaborado a partir dos dados de INMET (2019).

Caracterizando e analisando as secas durante o período de 1987-2018, bem como nas décadas, dentre os impactos que podem ser verificados referemse à produtividade agrícola do milho no ano de 2012, cultura esta característica no município de Serrinha-BA. A seca foi caracterizada em meteorológica, agrícola e hidrológica, o balanço hídrico de cultivo registrou produtividade final correspondente a $7,94 \%$ e perda total decorrente da deficiência hídrica em 92,06\% (IBGE, 2018; INMET, 2019). Segundo dados da produção agrícola municipal (IBGE, 2018), no ano de 2012 a quantidade de área plantada para milhos em grãos (duas safras) foi equivalente a 4000 ha, no entanto, a colheita, a quantidade produzida e o rendimento médio foi zero.

Associando os dados do município aos do semiárido estudados por Marengo, Cunha, Alves (2016) no período entre 2011 a 2014, pode-se observar uma relação entre si, pois apresentaram uma grande área com balanço hídrico negativo em quase toda região semiárida desde o centro do sul da BA até o RN, CE e PI.

Esta questão também pode ser confirmada com pesquisas de Martins e Magalhães (2015) em que informam ser 2012 e 2013 anos muito secos no Nordeste, conduzindo a elevadas quedas na produção de todos os tipos de 
lavouras temporárias, dentre elas milho e feijão, assim como observado no município de Serrinha.

Para realizar esta mesma comparação com o ano de 2014, caracterizado pela seca hidrológica, a produtividade foi de $17,53 \%$ e perda de $82,47 \%$. Neste ano, houve, para a cultura de milho, a quantidade de 4000 há de área plantada, 1680 ton produzidas, com rendimento médio de $420 \mathrm{Kg}$ por ha. Como consequência destes fatores, segundo dados do IBGE (2018), entre 2015 e 2016 em decorrência à estiagem prolongada, todas as principais culturas do estado tiveram queda na produção, o milho cerca de $40 \%$ e feijão, $60 \%$.

No ano de 2017, em que a seca foi caracterizada em meteorológica e agrícola no município de Serrinha, variando de severa a extrema, a produtividade do milho foi de $20,7 \%$, com perda de $79,22 \%$. Aqui a área plantada foi o equivalente a 4564 ha, 1337 ha de área colhida, produção de 310 toneladas e rendimento médio de $232 \mathrm{~kg}$ por ha (IBGE, 2018; INMET, 2019).

Assim, os tipos de secas e seus comportamentos são estratégias de suma importância na realização de um planejamento agrícola, em que se podem verificar regiões e localidades com potencialidades segundo os cultivos específicos. E também oportunizar a segurança hídrica, garantindo as cinco dimensões da água: beber, uso doméstico, agricultura, emergência e para o meio ambiente (SCHISTEK, 2000).

\section{CONSIDERAÇÕES FINAIS}

A quantidade de eventos secos foi maior em contraposição aos extremos. No entanto, sua categoria, duração e frequência foram sendo modificadas segundo o SPI aplicado. Com as décadas de 1990 e 2010 apresentando maior percentual de eventos secos em comparação à década de 2000, que por sua vez apresentou um maior registro de eventos úmidos.

Os anos de 1993 e 2012, considerados os mais secos da série histórica estudada e caracterizados por secas meteorológica, agrícola e hidrológica, reforçam a necessidade de analisar as consequências socioambientais no município de Serrinha-BA, o qual também está passando pelos efeitos das mudanças climáticas, visto que os anos mais secos estão mais recorrentes, ocorrendo a redução do índice pluviômetro, ampliando a deficiência hídrica e afetando a economia local.

Desta forma, o município de Serrinha-BA que tem a agricultura familiar como uma das bases econômicas e que utiliza tecnologias voltadas à captação da água de chuva nos processos produtivos, pesquisas como esta que possibilitam conhecer a dinâmica das secas e como estas vêm se comportando ao longo do tempo, podem ser utilizadas de forma a auxiliar no planejamento para a tomada de decisões futuras que fomentem políticas públicas voltadas à convivência com as secas e o desenvolvimento local.

\section{REFERÊNCIAS}

ABRAMOWITZ, M.; STEGUN, I. A. Handbook of Mathematical Functions with formulas, graphs, and mathematical tables. New York: Dover Publications Inc., $1965.1046 \mathrm{p}$. 
BARBOSA, H. A.; LAKSMI KUMAR, T. V. Influence of rainfall variability on the vegetation dynamics over Northeastern Brazil. Journal of Arid Environments, London, $\quad v$. 124, p. 377-387, $2016 . \quad$ DOI: https://doi.org/10.1016/j.jaridenv.2015.08.015

BHALME, H. N.; MOOLEY, D. A. Large-scale drought/floods and monsoon circulation. Monthly Weather Review, Boston, v. 108, n. 8, p. 1197-1211, 1980.

BLAIN, G. C.; BRUNINI, O. Avaliação e adaptação do Índice de Severidade de Seca de Palmer (PDSI) e do Índice Padronizado de Precipitação (SPI) às condições climáticas do Estado de São Paulo. Bragantia, Campinas, v. 64, n. 4, p.695-705, 2005. DOI: https://doi.org/10.1590/S0006-87052005000400020

BURITI, C. O.; BARBOSA, H. A. Um século de secas: por que as políticas hídricas não transformaram o semiárido brasileiro? São Paulo: Chiado Books, 2018.

CAMPOS, J. N. Secas e políticas públicas no semiárido: ideias, pensadores e períodos. Estudos Avançados, São Paulo, v. 28, n. 82, p. 65-88, 2014.

CAMURÇA, C. E. S.; ALENCAR, A. B.; CIDADE, E. C.; XIMENES, V. M. Implicações psicossociais da seca na vida de moradores de um município da zona rural do nordeste do Brasil. Avances en Psicología Latinoamericana, Bogotá, v. 34, n. 1, p. 117-128, 2016. DOI: dx.doi.org/10.12804/apl34.1.2016.08

CHAO, N.; WANG, Z.; JIANG, W.; CHAO, D. A quantitative approach for hydrological drought characterization in southwestern China using GRACE. Hydrogeology Journal, v. 24, p. 893-903, 2016.

COSTA, J. A. O fenômeno El NIÑO e as secas no Nordeste do Brasil. Revista Científica do IFAL, Maceió, v. 3, n. 1, p. 71-82, 2012.

CUNHA, A. P. M. A.; TOMASELLA, J.; RIBEIRO-NETO, G. G.; BROWN, M.; GARCIA,S. R.; BRITO, S. B.; CARVALHO, M. A. Changes in the spatial-temporal patterns of droughts in the Brazilian Northeast. Atmospheric Science Letters, v. 19, n. 10, p. e855, 2018. DOI: https://doi.org/10.1002/asl.855

EDWARDS, D. C.; MCKEE, T. B. Characteristics of 20th century drought in the United States at multiple time scales. Climatology Report, Colorado, n. 97-2, 1997. (Atmospheric Science Paper, 634).

FARIAS, A. A.; SOUZA, J. T. A. SOUSA, F. A. S. Identificação e análise de secas severas e extremas no município de Taperoá-PB. Revista Brasileira de Geografia Física, Recife, v. 7, n. 5, p. 818-826, 2014.

GUEDES, R. V. S.; SOUSA, F. A. S.; SILVA, F. D. S.; SILVA, V. P. R. Identificação e classificação espaço-temporal de eventos críticos chuvosos ou secos ocorridos em Pernambuco. Ciência e Natura, Santa Maria, v. 38 n. 1, p. 413-428, 2016.

HAIED, N.; FOUFOU, A.; CHAAB, S.; AZLAOUI, M.; KHADRI, S.; BENZAHIA, K.; BENZAHIA, I. Drought assessment and monitoring using meteorological indices in a semi-arida region. Energy Procedia, v. 119, p. 518-529, 2017. DOI: $10.1016 /$ j.egypro.2017.07.064

HAILE, G. G.; TANG, Q.; LENG, G.; JIA, G.; WANG, J.; CAI, D.; SUN, S.; BANIYA, B.; ZHANG, Q. Long-term spatiotemporal variation of drought patterns 
over the Greater Horn of Africa. Science of the Total Environment, Amsterdam, v. 704, n. 135299, 2020. DOI: https://doi.org/10.1016/j.scitotenv.2019.135299

HAILE, G. G.; Tang, Q.; SUN, S.; Huang, Z.; Zhang, X.; LIU, X. Droughts in East Africa: causes, impacts and resilience. Earth-Science Reviews, Amsterdam, v. 193, p. 146-161, 2019. DOI: https://doi.org/10.1016/j.earscirev.2019.04.015

HAMEED, M.; AHMADALIPOUR, A.; MORADKHANI, H. Drought and food security in the middle east: an analytical framework. Agricultural and Forest Meteorology, Amsterdam, v. 281, 107816, 2020. DOI:

https://doi.org/10.1016/j.agrformet.2019.107816

HAYES, M.; SVOBODA, M.; WALL, N.; WIDHALM, M. The Lincoln declaration on drought indices. Universal meteorological drought index recommended. Bulletin of the American Meteorological Society, Boston, v. 92, n. 4, p. 485-488, 2011.

IBGE. Instituto Brasileiro de Geografia e Estatística. Sinopse do censo demográfico 2010. Rio de Janeiro: IBGE, 2010. Disponível em: http://www.censo2010.ibge.gov. br/sinopse. Acesso em: 12 ago. 2019.

IBGE. Instituto Brasileiro de Geografia e Estatística. Produção Agrícola Municipal. PAM 2018. Disponível em: https://sidra.ibge.gov.br/acervo\#/S/PA/A/23/T/Q. Acesso em: 27 abr. 2020.

INMET. Instituto Nacional de Meteorologia. Disponível em: http://www.inmet.gov.br/portal/index.php?r=bdmep/bdmep. Acesso em: 15 abr. 2019.

KEYANTASH, J.; DRACUP, J. The quantification of drought: an evaluation of drought indices. Bulletin of the American Meteorological Society, Boston, v. 83, n. 8, p. 1167-1180, 2002. DOI: https://doi.org/10.1175/1520-0477-83.8.1167

LEIVAS, J. F.; ANDRADE, R. G.; VICTORIA, D. C.; TORRESAN, F. E.; BOLFE, E. L. Monitoramento da seca $2011 / 2012$ no nordeste brasileiro a partir do satélite SPOT vegetation e TRMM. Engenharia na agricultura, Viçosa, v. 22, n. 3, p. 211221, 2014.

LONITA, M.; SCHOLZ, P.; CHELCEA, S. Assessment of droughts in Romania using the Standardized Precipitation Index. Natural Hazards, Dordrecht, v. 81 , p. $1483-1498,2016$. DOI: https://doi.org/10.1007/s11069-015-2141-8.

MACEDO, M. J. H.; GUEDES, R. V. S.; SOUSA, F. A. S. Monitoramento e intensidade das secas e chuvas na cidade de Campina Grande/PB. Revista Brasileira de Climatologia, Curitiba, v. 8, n. 7, p. 105-117, 2011. DOI: http://dx.doi.org/10.5380/abclima.v8i0.25797

MARENGO, J. A.; CUNHA, A. P.; ALVES, L. M. A. A seca de 2012-15 no semiárido do Nordeste do Brasil no contexto histórico. Revista Climanálise, Cachoeira Paulista, v. 3, p. 49-54, 2016.

MARTINS, E. S. P. R.; MAGALHAES, A. R. A seca de 2012-2015 no Nordeste e seus impactos. Parcerias Estratégicas, Brasília, ed. esp., v. 20, n. 41, p. 107$128,2015$.

MATOS, M. P. S. R. Famílias desagregadas sobre a terra ressequida: indústria da seca e deslocamentos familiares no nordeste do Brasil. Nómadas. Revista Crítica 
de CienciasSociales y Jurídicas, Barcelona, número especial, 2012. DOI: https://doi.org/10.5209/rev_NOMA.2012.41773

MCKEE, T. B.; DOESKEN, N. J.; KLEIST, J. The relationship of drought frequency and duration of time scales. Eighth Conference on Applied Climatology. American Meteorological Society, Boston, p. 179-184, 1993.

MISHRA, A. K.; SINGH, V. P. A review of drought concepts. Journal of Hydrology, Amsterdam, v. 391, n. 1-2, p. 202-216, 2010.DOI: https://doi.org/10.1016/j.jhydrol.2010.07.012

MONITOR DE SECAS. Tabela de Classificação de Severidade das Secas. Disponível em http://monitordesecas.ana.gov.br/tabela-de-classificacao. Acesso em 09 mar 2021

NASCIMENTO, F. C. A.; BRAGA, C. C.; ARAÚJO, F. R. C. D. Análise estatística dos eventos secos e chuvosos de precipitação do Estado do Maranhão. Revista Brasileira de Meteorologia, São José dos Campos, v. 32, n. 3, p. 375-386, 2017.

OLORUNTADE, A. J.; MOHAMMAD, T. A.; GHAZALI, A. H.; WAYAYOK, A. Analysis of meteorological and hydrological droughts in the Niger-South Basin, Nigeria. Global and Planetary Change, Amsterdam, v. 155, p. 225-233, 2017. DOI: 10.1016/j.gloplacha.2017.05.002

PALMER, W. C. Meteorological drought. Washington, 1965. (Research Paper, 45).

PTDSS. Plano de Desenvolvimento Territorial Sustentável e Solidário do Território do Sisal. Núcleo de Extensão em Desenvolvimento Territorial. Valente: Universidade Estadual de Feira de Santana, 2016.

RAVELO, A.; SANZ RAMOS, R.; DOURIET CÁRDENAS, J. Detección, evaluación y pronóstico de las sequias em la región del Organismo de Cuenca Pacífico Norte, México. AgriScientia, Cordoba, v. 31, n. 1, p. 11-24. 2014. DOI: https://doi.org/10.31047/1668.298x.v31.n1.9836

SALVADOR, C.; NIETO, R.; LINARES, C.; DÍAZ, J.; GIMENOA, L. Effects of droughts on health: diagnosis, repercussion, and adaptation in vulnerable regions under climate change. Challenges for future research. Science of the Total Environment, Amsterdam, v. 703, n. 134912, 2020. DOI: https://doi.org/10.1016/j.scitotenv.2019.134912

SANTOS, T. C. C.; CÂMARA, J. B. D. (orgs.). Geo Brasil 2002 - Perspectivas do meio ambiente no Brasil. Edições IBAMA: Brasília, 2002.

SCHISTEK, H. A Água no Semiárido Brasileiro. E-book. 2000. Disponível: https://irpaa.org/ebookbr/page12.htm

SEI-BA. Superintendência de Estudos Econômicos e Sociais da Bahia. Uso atual das terras: bacias do Recôncavo Norte e do Rio Inhambupe. Salvador: SEI-BA, 2003. (Série estudos e pesquisas, 64).

SEI-BA. Superintendência de Estudos Econômicos e Sociais da Bahia. Cartografia Temática - regionalizações - conteúdo geral - arquivos vetoriais. Disponível em: http://www.sei.ba.gov.br/index.php?option=com_content\&view=article\&id $=266$ o\&Itemid=667. Acesso em: 16 maio 2019. 
SEI-BA. Superintendência de Estudos Econômicos e Sociais da Bahia. Indicadores municipais.

http://www.sei.ba.gov.br/site/resumos/indicadores/indicadores_2930501.pdf.

Acesso em: 28 fev. 2021.

SILVA, A.; SANTO, F.; PIRES, V. A seca em Portugal: prevenção, monitorização e mitigação. 2005. Disponível em: http://panda. igeo.pt/pancd/pdfs/seca2005/FatimaEspiritoSanto.pdf. Acesso em:

10 set. 2019.

SOUZA, A. G. S. S.; RIBEIRO NETO, A.;ROSSATO, L.; ALVALÁ, R. C. S.; SOUZA, L. L. Use of SMOS L3 Soil Moisture Data: Validation and Drought Assessment for Pernambuco State, Northeast Brazil. Remote Sensing, Basel, v. 10, n. 8, p. 1314, 2018. DOI: https://doi.org/10.3390/rs10081314

SUBASH, N.; MOHAN, H. S. R. Trend detection in rainfall and evaluation of standardized precipitation index as a drought assessment index for rice-wheat productivity over IGR in India. International Journal of Climatology, Chichester, v. 31 p. 1694-1709, 2011.

SUN, F.; MEJIA, A.; ZENG, P.; CHE, Y. Projecting meteorological, hydrological and agricultural droughts for the Yangtze River basin. Science of the Total Environment, Amsterdam, v. 696, n. 134076, 2019. DOI: https://doi.org/10.1016/j.scitotenv.2019.134076

VAN-ROOY, M. P. A Rainfall Anomaly Index (RAI), independent of time and space. Notos, Petroria, v. 14, p. 43-48, 1965.

WEGHORST, K. M. The reclamation drought index: guidelines and practical applications. Denver: Bureau of Reclamation, 1996 